\title{
Nonlocal conditions for differential inclusions in the space of functions of bounded variations
}

\author{
Ravi Agarwal ${ }^{1,2}$ and Abdelkader Boucherif $2^{2^{*}}$
}

* Correspondence:

aboucher@kfupm.edu.sa

${ }^{2}$ Department of Mathematics and

Statistics, King Fahd University of

Petroleum and Minerals, Box 5046,

Dhahran 31261, Saudi Arabia

Full list of author information is

available at the end of the article

\section{Abstract}

We discuss the existence of solutions of an abstract differential inclusion, with a right-hand side of bounded variation and subject to a nonlocal initial condition of integral type.

\section{AMS Subject Classification}

34A60, 34G20, 26A45, 54C65, 28B20

Keywords: Set-valued maps of bounded variation, Differential inclusion, Nonlocal initial condition, Generalized Helly selection principle, Fixed point of multivalued operators

\section{Introduction}

Solutions of differential equations with smooth enough coefficients cannot have jump discontinuities, see for instance $[1,2]$. The situation is quite different for systems described by differential equations with discontinuous right-hand sides [3]. Examples of such systems are mechanical systems subjected to dry or Coulomb frictions [4], optimal control problems where the control parameters are discontinuous functions of the state [5], impulsive differential equations [6], measure differential equations, pulse frequency modulation systems or models for biological neural nets [7]. For these systems the state variables undergo sudden changes at their points of discontinuity. The mathematical models of many of these systems are described by multivalued differential equations or differential inclusions [8].

Let $X$ be a Banach space with norm $|\cdot|_{X}$. Then $X$ is a metric space with the distance $d_{X}$ defined by

$$
d_{X}(x, y)=|x-y|_{X^{\prime}} \text { for any } x, y \in X .
$$

Let $I=[0, T]$ be a compact real interval. We are interested in the study of the following multivalued nonlocal initial value problem

$$
\left\{\begin{array}{l}
\dot{x}(t) \in F(t, x(t)), t \in I \\
x(0+)=\int_{0}^{T} g(x(t)) d t
\end{array}\right.
$$

where $F: I \times X \rightarrow X$ is a multivalued map and $g: X \rightarrow X$ is continuous.

\section{SpringerOpen ${ }^{\circ}$}

(c) 2011 Agarwal and Boucherif; licensee Springer. This is an Open Access article distributed under the terms of the Creative Commons Attribution License (http://creativecommons.org/licenses/by/2.0), which permits unrestricted use, distribution, and reproduction in any medium, provided the original work is properly cited. 
The investigation of systems subjected to nonlocal conditions started with [9] for partial differential equations and [10] for Sturm-Liouville problems. For more recent work we refer the interested reader to [11] and the references therein.

It is clear that solutions of (1) are solutions of the integral inclusion

$$
x(t) \in \int_{0}^{T} g(x(t)) d t+\int_{0}^{t} F(s, x(s)) d s .
$$

\section{Preliminaries}

Definition 1 We say that $f: I \rightarrow X$ is of bounded variation, and we write $f \in B V$ $(I, X)$, if

$$
V_{d_{X}}(f, I)=\sup _{\Pi} \sum_{i=1}^{m} d_{X}\left(f\left(\tau_{i}\right), f\left(\tau_{i-1}\right)\right)<+\infty,
$$

where $\Pi: \tau_{0}=0<\tau_{1}<\ldots<\tau_{m}=T$ is any partition of $I$. The quantity $V_{d_{X}}(f, I)$ is called the total variation of $f$.

We shall denote by $\mathrm{BV}(I, X)$ the space of all functions of bounded variations on $I$ and with values in $X$. It is a Banach space with the norm $|\cdot|_{b}$ given by

$$
|f|_{b}=|f(0+)|_{X}+V_{d_{X}}(f, I) \text {, for any } f \in \operatorname{BV}(I, X) .
$$

In order to discuss the integral inclusion (2) we present some facts from set-valued analysis. Complete details can be found in the books $[8,12,13]$. Let $\left(X,|\cdot|_{X}\right)$ and $\left(Y,|\cdot|_{Y}\right)$ be Banach spaces. We shall denote the set of all nonempty subsets of $X$ having property $\ell$ by $\wp_{\ell}(X)$. For instance, $A \in \wp_{c} \ell(X)$ means $A$ closed in $X$, when $\ell=b$ we have the bounded subsets of $X, \ell=\mathrm{cv}$ for convex subsets, $\ell=\mathrm{cp}$ for compact subsets and $\ell$ $=\mathrm{cp}, \mathrm{cv}$ for compact and convex subsets. The domain of a multivalued map $\mathfrak{R}: X \rightarrow Y$ is the set $\operatorname{dom} \mathfrak{R}=\{z \in X ; \mathfrak{R}(z) \neq \varnothing\}$. $\mathfrak{R}$ is convex (closed) valued if $\mathfrak{R}(z)$ is convex (closed) for each $z \in X: \Re$ has compact values if $\Re(z) \in \wp_{\mathrm{cv}}(Y)$ for every $z \in X$; $\mathfrak{R}$ is bounded on bounded sets if $\mathfrak{R}(A)=\mathrm{U}_{z \in A} \Re(z)$ is bounded in $Y$ for all $A \in \wp_{b}(X)$ (i.e. $\left.\sup _{z \in A}\left\{\sup \left\{|y|_{Y} ; y \in \mathfrak{R}(z)\right\}\right\}<\infty\right): \mathfrak{R}$ is called upper semicontinuous (u.s.c.) on $X$ if for each $z \in X$ the set $\mathfrak{R}(z) \in \wp_{c l}(Y)$ is nonempty, and for each open subset $\Lambda$ of $Y$ containing $\Re(z)$, there exists an open neighborhood $\Pi$ of $z$ such that $\Re(\Pi) \subset \Lambda$. In terms of sequences, $\Re$ is u.s.c. if for each sequence $\left(z_{n}\right) \subset X, z_{n} \rightarrow z_{0}$, and $B$ a closed subset of $Y$ such that $\mathfrak{R}\left(z_{n}\right) \cap B \neq \varnothing$, then $\mathfrak{R}\left(z_{0}\right) \cap B \neq \varnothing$. The set-valued map $\mathfrak{R}$ is called completely continuous if $\mathfrak{R}(A)$ is relatively compact in $Y$ for every $A \in \wp(X)$. If $\Re$ is completely continuous with nonempty compact values, then $\Re$ is u.s.c. if and only if $\mathfrak{R}$ has a closed graph (i.e. $z_{n} \rightarrow z, w_{n} \rightarrow w, w_{n} \in \mathfrak{R}\left(z_{n}\right) \Rightarrow w \in \mathfrak{R}(z)$ ). When $X \subset Y$ then $\Re$ has a fixed point if there exists $z \in X$ such $z \in \mathfrak{R}(z)$. A multivalued map $\mathfrak{R}: J \rightarrow \wp_{c l}$ $(X)$ is called measurable if for every $x \in X$, the function $\theta: J \rightarrow \mathbb{R}$ defined by $\theta(t)=$ $\operatorname{dist}(x, \mathfrak{R}(t))=\inf \left\{|x-z|_{X} ; z \in \mathfrak{R}(t)\right\}$ is measurable. $|\mathfrak{R}(z)|_{Y}$ denotes $\sup \left\{|y|_{Y} ; y \in \mathfrak{R}(z)\right\}$.

If $A$ and $B$ are two subsets of $X$, equipped with the metric $d_{X}$, such that $d_{X}(x, y)=\mid x$ - $\left.y\right|_{X}$, the Hausdorff distance between $A$ and $B$ is defined by

$$
d_{H}(A, B)=\max \{\rho(A, B), \rho(B, A)\},
$$


Where

$$
\rho(A, B)=\sup _{a \in A} d_{X}(a, B), \text { and } d_{X}(a, B)=\inf _{b \in B} d_{X}(a, b) .
$$

It is well known that $\left(\wp_{b, c l}(X), d_{H}\right)$ is a metric space and so is $\left(\wp_{c p}(X), d_{H}\right)$.

Definition 2 (See $[14,15]) \Theta: I \rightarrow X$ is of bounded variation (with respect to $d_{H}$ ) on $I$ if

$$
V(\Theta, I)=V_{d_{H}}(\Theta, I)=\sup _{\Pi}\left[\sum_{i=1}^{m} d_{H}\left(\Theta\left(t_{i}\right), \Theta\left(t_{i-1}\right)\right]<\infty,\right.
$$

where the supremum is taken over all partitions $\Pi=\left\{t_{i} ; i=1,2, \ldots, m\right\}$ of the interval I.

Definition 3 Let $X^{I}$ denote the set of all functions from I into X. The Nemitskii (or superposition) operator corresponding to $F: I \times X \rightarrow X$ is the operator

$$
N_{F}: X^{I} \rightarrow X,
$$

defined by

$$
N_{F}(x)(t)=F(t, x(t)) \text { for every } t \in I .
$$

Definition 4 The multifunction $F: I X \rightarrow X$ is of bounded variation if for any function $\times \in \operatorname{BV}(I, X)$ the multivalued map $N_{F}(x): I \rightarrow X$ is of bounded variation on I (in the sense of Definition 2) and

$$
V_{d_{H}}(F(\cdot, x(\cdot)), I)=V_{d_{H}}\left(N_{F}(x), I\right) \text {. }
$$

Definition 5 Let $\Delta$ be a subset of $I \times X$. We say that $\Delta$ is $\mathcal{L} \otimes \mathcal{B}$ measurable if $\Delta$ belongs to the $\sigma$-algebra generated by all sets of the form $J \times D$ where $J$ is Lebesgue measurable in I and D is Borel measurable in $X$.

Theorem 6 (Generalized Helly selection principle) [[14], Theorem 5.1 p. 812] Let K be a compact subset of the Banach space $\times$ and let $\mathcal{F}$ be a family of maps of uniformly bounded variation from $I$ into $K$. Then there exists a sequence of maps $\left(f_{n}\right)_{n \geq 1} \subset \mathcal{F}$ convergent pointwise on $I$ to a map $f: I \rightarrow K$ of bounded variation such that $V(f, I) \leq \sup _{\varphi \in \mathcal{F}} V(\varphi, I)$.

In the next theorem we shall denote by $\bar{U}$ and $\partial U$ the closure and the boundary of a set $U$.

Theorem 7 ([[16], Theorem 3.4, p. 34]) Let $U$ be an open subset of a Banach space $Z$ with $0 \in U$. Let $A: \bar{U} \rightarrow Z$ be a single-valued operator and $B: \bar{U} \rightarrow \wp_{\mathrm{cp}, \mathrm{cv}}(Z)$ be a multivalued operator such that

(i) $A(\bar{U})+B(\bar{U})$ is bounded,

(ii) $A$ is a contraction with constant $k \in(0,1 / 2)$,

(iii) $B$ is u.s.c and compact.

Then either

(a) the operator inclusion $\lambda x \in A x+B x$ has a solution for $\lambda=1$, or

(b) there is an element $u \in \partial U$ such that $\lambda u \in A u+B u$ for some $\lambda>1$. 


\section{Main results}

In this section we state and prove our main result. We should point out that no semicontinuity property is assumed on the multifunction $F$, which is usually the case in the literature. We refer the interested reader to the nice collection of papers in [17] and the references therein.

Theorem 8 Assume that the following conditions hold.

(H1) $g: X \rightarrow X$ is continuous, $g(0)=0$ and there exists $\theta:[0,+\infty) \rightarrow[0,+\infty)$ continuous and $\theta(r) \leq \beta r$, with $\beta<1 / 2$ and $\beta T \neq 1$, such that

$$
|g(u)-g(v)|_{X}<\theta\left(|u-v|_{X}\right),
$$

(H2) $F: I \times X \rightarrow \wp_{\mathrm{cp}, \mathrm{cv}}(X)$ is of bounded variation such that

(i) $(t, x) \mapsto F(t, x)$ is $\mathcal{L} \otimes \mathcal{B}$ measurable,

(ii) there exists an integrable function $q: I \rightarrow[0,+\infty)$ with

$|F(t, x)|_{X} \leq q(t)$ for $(t, x) \in I \times X$,

(iii) $x_{k} \rightarrow x$ as $k \rightarrow \infty$ pointwise implies $d_{H}\left(F\left(t, x_{k}\right), F(t, x)\right) \rightarrow 0, k \rightarrow \infty$.

Then problem (1) has at least one solution in $\operatorname{BV}(I, X)$.

Proof. Let $Q=\sup _{t \in I} \int_{0}^{t} q(s) d s$. We show that there exists $M>0$ such that all possible solutions of (2) in $\mathrm{BV}(I, X)$, satisfy

$$
|x|_{b} \leq M \text {. }
$$

Recall that solutions of (1) satisfy

$$
x(t) \in \int_{0}^{T} g(x(t)) d t+\int_{0}^{t} F(s, x(s)) d s=\int_{0}^{T} g(x(t)) d t+\int_{0}^{t} N_{F}(x)(s) d s .
$$

Since the multivalued map $N_{F}(x): I \rightarrow X$ is of bounded variation it admits a selector $f$ $: I \rightarrow X$ of bounded variation such that

$$
V_{d_{X}}(f, I) \leq V_{d_{H}}\left(N_{F}(x), I\right),
$$

see [[18], Theorem A, p. 250].

It follows from (3) that

$$
x(t)=\int_{0}^{T} g(x(t)) d t+\int_{0}^{t} f(s) d s, t \in I .
$$

This implies

$$
\begin{aligned}
|x(t)|_{X} & \leq\left|\int_{0}^{T} g(x(t)) d t\right|+\left|\int_{0}^{t} f(s) d s\right|_{X} \\
& \leq \int_{0}^{T}|g(x(t))|_{X} d t+\int_{0}^{t}|f(s)|_{X} d s .
\end{aligned}
$$


The condition on $g$ and (H2) (ii) imply

$$
|x(t)|_{X} \leq \beta \int_{0}^{T}|x(t)|_{X} d t+\int_{0}^{t} q(s) d s .
$$

Hence

$$
\int_{0}^{T}|x(t)|_{X} d t \leq \beta T \int_{0}^{T}|x(t)|_{X} d t+\int_{0}^{T} \int_{0}^{t} q(s) d s d t .
$$

This last inequality yields

$$
\int_{0}^{T}|x(t)|_{X} d t \leq \frac{1}{1-\beta T} \int_{0}^{T} \int_{0}^{t} q(s) d s d t .
$$

Since

$$
\int_{0}^{T} \int_{0}^{t} q(s) d s d t=\int_{0}^{T}(T-s) q(s) d s,
$$

we obtain

$$
\int_{0}^{T}|x(t)|_{X} d t \leq \frac{1}{1-\beta T} \int_{0}^{T}(T-s) q(s) d s,
$$

so that

$$
\int_{0}^{T}|x(t)|_{X} d t \leq \frac{2 T}{1-\beta T} Q .
$$

Inequality (5) and the condition on $g$ imply that

$$
\int_{0}^{T}|g(x(t))|_{X} d t \leq \frac{2 \beta T}{1-\beta T} Q .
$$

Hence any possible solution $x$ of (2) in BV(I,X), satisfies

$$
|x(0+)|_{X} \leq \frac{2 \beta T}{1-\beta T} Q .
$$

Let $\Pi=\left\{t_{i j} ; i=1,2, \ldots, m\right\}$ be any partition of the interval $I$, and let $x \in \operatorname{BV}(I, X)$ be any possible solution of (2). It follows from (4) that

$$
x\left(t_{i}\right)-x\left(t_{i-1}\right)=\int_{t_{i-1}}^{t_{i}} f(s) d s, i=1, \ldots, m .
$$

It is easily shown that

$$
V_{d_{X}}(x, I) \leq V_{d_{X}}(f, I) \leq \sup _{\Pi}\left[\sum_{i=1}^{m} \int_{\tau_{i-1}}^{\tau_{i}} q(s) d s\right] \leq Q .
$$


Therefore

$$
|x|_{b} \leq \frac{2 \beta T}{1-\beta T} Q+Q
$$

Letting $M:=\frac{1+\beta T}{1-\beta T} Q$, we see that

$$
|x|_{b} \leq M \text {. }
$$

Let

$$
\Omega:=\left\{x \in \operatorname{BV}(I, X) ;|x|_{b}<M+1\right\} .
$$

Define two operators

$$
A: \Omega \rightarrow X, B: \Omega \rightarrow X
$$

by

$$
A x(t)=\int_{0}^{T} g(x(t)) d t,
$$

and

$$
B x(t)=\int_{0}^{t} F(s, x(s)) d s=\int_{0}^{t} N_{F}(x)(s) d s .
$$

First, we show that $A(\bar{\Omega})+B(\bar{\Omega})$ is bounded, i.e. $\sup _{x \in \bar{\Omega}}\left\{\sup \left\{|y|_{b^{\prime}} ; y \in A(x)+B(x)\right\}\right\}<\infty$.

Let $y \in A(\bar{\Omega})+B(\bar{\Omega})$. Then there exists $x \in \bar{\Omega}$ such that

$$
y \in A(x)+B(x) .
$$

It follows from (3) that $|y|_{b} \leq M$.

(H1) implies that the single-valued operator $A$ is a contraction with constant $k \in(0$, $1 / 2)$.

Claim 1. The multivalued operator $B$ has compact and convex values. For, since $F: I$ $\times X \rightarrow \wp_{\mathrm{cp}, \mathrm{cv}}(X)$ it follows that $N F: X^{I} \rightarrow \wp_{\mathrm{cp}, \mathrm{cv}}(X)$, i.e. has compact and convex values. This implies that the Aumann integral

$$
\int_{0}^{t} N_{F}(x)(s) d s
$$

has compact and convex values. See for instance [5].

Claim 2. $B$ is completely continuous, i.e. $B(\Omega)$ is a relatively compact subset of $B V(I$, $X)$. Let $q \in \Omega$ be arbitrary. Then for every $f \in N_{F}(q)$ the function $u: I \rightarrow X$ defined by

$$
u(t)=\int_{0}^{t} f(s) d s,
$$

satisfies

$$
\dot{u}(t)=f(t), \quad \mathrm{u}(0+)=0 .
$$


If we write

$$
u=\Upsilon f,
$$

then the operator $\Upsilon: X \rightarrow X$ is continuous and

$$
B=\Upsilon \circ N_{F} .
$$

Let $\left(B x_{k}\right)_{k \geq 1}$ be a sequence in $B(\Omega)$. Then the sequence $\left(x_{k}\right)_{k \geq 1}$ is uniformly bounded and is of bounded variation. Theorem 4 shows that there exists a subsequence, which we label the same, and which converges pointwise to $y \in \Omega$. We have

$$
\left|B x_{k}-B y\right|_{b} \leq \sup _{\Pi}\left[\sum_{i=1}^{m} \int_{\tau_{i-1}}^{\tau_{i}}\left|F\left(s, x_{k}(s)\right)-F(s, y(s))\right|_{X} d s\right] .
$$

Assumption (H2) (iii) implies that

$$
\left|B x_{k}-B y\right|_{b} \rightarrow 0 \text { as } k \rightarrow 0 .
$$

This proves the claim.

Claim 3. $B$ is u.s.c. Since $B$ is completely continuous it is enough to show that its graph is closed. Let $\left\{\left(x_{n}, y_{n}\right)\right\}_{n \geq 1}$ be a sequence in $\operatorname{graph}(B)$ and let $(x, y)=\lim _{n \rightarrow \infty}\left(x_{n}\right.$, $\left.y_{n}\right)$. Then $y_{n} \in B\left(x_{n}\right)$, i.e $y_{n}(t) \in \int_{0}^{t} F\left(s, x_{n}(s)\right) d s, t \in I$. This implies that

$$
y_{n}(t) \in \int_{0}^{t} F(s, x(s)) d s+\int_{0}^{t}\left[F\left(s, x_{n}(s)\right)-F(s, x(s))\right] d s .
$$

Since $x_{n} \rightarrow x$ in $X$ it follows from (H2)(ii) that

$$
\lim _{n \rightarrow \infty} y_{n}(t) \in \int_{0}^{t} F(s, x(s)) d s
$$

which shows that

$$
y \in B(x) .
$$

Hence $(x, y) \in \operatorname{graph}(B)$, and $B$ has a closed graph.

Finally, alternative (b) in Theorem 5 cannot hold due to (3) and the choice of $\Omega$.

By Theorem 5 the inclusion

$$
x \in A x+B x,
$$

has at least one solution in $\operatorname{BV}(I, X)$. This completes the proof of the theorem.

For our second result we consider the case when $\int_{0}^{T} g(x(t)) d t=\int_{0}^{T} \psi(t) x(t) d t$, where $\psi: I \rightarrow \mathbb{R}$ is continuous. Let

$$
\psi_{0}=\int_{0}^{T} \psi(t) d t \text { and } \lambda(\mathrm{s})=\int_{\mathrm{s}}^{\mathrm{T}} \frac{\psi(\mathrm{t}) \mathrm{dt}}{1-\psi_{0}} .
$$

From the definition of the function $\lambda$ we infer that, if $\psi^{*}=\max _{t \in I}|\psi(t)|$,

$$
|\lambda(s)| \leq \frac{2 T}{1-\psi_{0}} \psi^{*} \text { for any } s \in I .
$$


Theorem 9 Assume that the following conditions hold

(H3) $\psi: I \rightarrow \mathbb{R}$ is continuous and $\psi_{0} \neq 1$,

(H4) $F: I \times X \rightarrow \wp_{\mathrm{cp}, \mathrm{cv}}(X)$ is of bounded variation such that

(i) $(t, x) \mapsto F(t, x)$ is $\mathcal{L} \otimes \mathcal{B}$ measurable,

(ii) there exists $\omega: I \times[0, \infty) \rightarrow(0, \infty)$ continuous, nondecreasing with respect to its second argument and

$$
\lim \sup _{\rho \rightarrow \infty} \frac{1}{\rho}\left(\frac{1-\psi_{0}+2 \psi^{*} T}{1-\psi_{0}}\right) \int_{0}^{T} \omega(s, \rho) d s<1,
$$

such that $|F(t, x)|_{X} \leq \omega \rightarrow\left(t,|x|_{b}\right)$.

(iii) $x_{k} \rightarrow x$ pointwise as $k \rightarrow \infty$ implies $d_{H}\left(F\left(t, x_{k}\right), F(t, x)\right) \rightarrow 0$ as $k \rightarrow \infty$.

Then problem (1) has at least one solution in $\operatorname{BV}(I, X)$.

Proof. Since the multivalued map $N_{F}(x): I \rightarrow X$ is of bounded variation it admits a selector $h: I \rightarrow X$ of bounded variation such that

$$
V_{d_{X}}(h, I) \leq V_{d_{H}}\left(N_{F}(x), I\right),
$$

see [[18], Theorem A, p. 250].

Solutions of (2) satisfy

$$
x(t)=x(0+)+\int_{0}^{t} h(s) d s, \quad h \in N_{F}(x) .
$$

Substituting the initial condition in (7) we obtain

$$
x(t)=\int_{0}^{T} \psi(t) x(t) d t+\int_{0}^{t} h(s) d s, \quad h \in N_{F}(x)
$$

Since $\psi_{0} \neq 1$ it follows that

$$
x(t)=\int_{0}^{T} \frac{\psi(t)}{1-\psi_{0}} \int_{0}^{t} h(s) d s d t+\int_{0}^{t} h(s) d s, \quad h \in N_{F}(x) .
$$

Thus, solutions of (2) are solutions of

$$
x(t)=\int_{0}^{T} \lambda(s) h(s) d s+\int_{0}^{t} h(s) d s, h \in N_{F}(x),
$$

and vice versa. It follows from (8)

$$
|x(t)|_{X} \leq \int_{0}^{T}|\lambda(s)| \omega\left(s,|x|_{b}\right) d s+\int_{0}^{t} \omega\left(s,|x|_{b}\right) d s .
$$

The upper bound on $|\lambda(s)|$ implies

$$
|x(t)|_{X} \leq \frac{2 T}{1-\psi_{0}} \psi^{*} \int_{0}^{T} \omega\left(s,|x|_{b}\right) d s+\int_{0}^{t} \omega\left(s,|x|_{b}\right) d s,
$$


which gives

$$
|x(0+)|_{X} \leq \frac{2 T}{1-\psi_{0}} \psi^{*} \int_{0}^{T} \omega\left(s,|x|_{b}\right) d s .
$$

Let $\Pi=\left\{t_{i} ; i=1,2, \ldots, m\right\}$ be any partition of the interval $I$, and let $x \in \operatorname{BV}(I, X)$ be any possible solution of (2). Then, it follows from (7) that

$$
x\left(t_{i}\right)-x\left(t_{i-1}\right)=\int_{t_{i-1}}^{t_{i}} h(s) d s, i=1, \ldots, m,
$$

which leads to

$$
V_{d_{X}}(x, I) \leq V_{d_{X}}(h, I) \leq \int_{0}^{T} \omega\left(s,|x|_{b}\right) d s .
$$

Since $|x|_{b}=|x(0+)|_{X}+V_{d_{X}}(x, I)$, we have

$$
|x|_{b} \leq \frac{2 T}{1-\psi_{0}} \psi^{*} \int_{0}^{T} \omega\left(s,|x|_{b}\right) d s+\int_{0}^{T} \omega\left(s,|x|_{b}\right) d s .
$$

Finally, we see that

$$
|x|_{b} \leq \frac{1-\psi_{0}+2 \psi^{*} T}{1-\psi_{0}} \int_{0}^{T} \omega\left(s,|x|_{b}\right) d s .
$$

Let

$$
\rho_{0}=|x|_{b} .
$$

Then (10) yields

$$
1 \leq \frac{1}{\rho_{0}}\left(\frac{1-\psi_{0}+2 \psi^{*} T}{1-\psi_{0}}\right) \int_{0}^{T} \omega\left(s, \rho_{0}\right) d s .
$$

The condition on the function $\omega$ implies that there exists $\rho^{*}>0$ such that for all $\rho>$ $\rho^{*}$

$$
\frac{1}{\rho}\left(\frac{1-\psi_{0}+2 \psi^{*} T}{1-\psi_{0}}\right) \int_{0}^{T} \omega(s, \rho) d s<1 .
$$

Comparing inequalities (11) and (12) we see that

$$
\rho_{0}=|x|_{b} \leq \rho^{*} .
$$

Let

$$
\Sigma=\left\{x \in \mathrm{BV}(I, X) ; \quad|x|_{b} \leq \rho^{*}\right\} .
$$

Then $\Sigma$ is nonempty, closed, bounded and convex.

Define a multivalued operator

$$
\digamma: \mathrm{BV}(I, X) \rightarrow \wp_{\mathrm{cp}, \mathrm{cv}}(X),
$$


by

$$
\digamma x(t)=\int_{0}^{T} \lambda(s) N_{F}(x)(s) d s+\int_{0}^{t} N_{F}(x)(s) d s .
$$

Then solutions of (2) are fixed point of the multivalued operator $\digamma: \Sigma \rightarrow \wp_{\mathrm{cp}, \mathrm{cv}}(X)$. It is clear that $\digamma(\Sigma) \subset \Sigma$. Proceeding as in the above claims we can show that $\digamma$ is u.s.c. and $\overline{\digamma(\Sigma)}$ is compact. By the Theorem of Bohnenblust and Karlin (see Corollary 11.3 in [8]) $\digamma$ has a fixed point in $\Sigma$, which is a solution of the inclusion (2), and therefore a solution of (1).

\section{Acknowledgements}

The authors are grateful to King Fahd University of Petroleum and Minerals for its constant support. The authors would like to thank an anonymous referee for his/her comments.

\section{Author details}

${ }^{1}$ Department of Mathematics, Florida Institute of Technology, Melbourne, FL, USA ${ }^{2}$ Department of Mathematics and Statistics, King Fahd University of Petroleum and Minerals, Box 5046, Dhahran 31261, Saudi Arabia

\section{Authors' contributions}

Both authors have read and approved the final manuscript.

\section{Competing interests}

The authors declare that they have no competing interests.

Received: 7 February 2011 Accepted: 24 June 2011 Published: 24 June 2011

\section{References}

1. Agarwal RP, O'Regan D: An Introduction to Ordinary Differential Equations. Universitext. Springer, New York; 2008

2. Coddington EA, Levinson N: Theory of Ordinary Differential Equations. McGraw-Hill Book Company, Inc., New York; 1955.

3. Filippov AF: Differential Equations with Discontinuous Righthand Sides. Kluwer Academic Publishers; 1988.

4. Deimling K: Multivalued differential equations and dry friction problems. In Delay and Differential Equations (Ames, IA, 1991). Edited by: Fink AM. World Scientific, River Edge, NJ; 1992:99-106.

5. Hermes H, LaSalle JP: Functional Analysis and Time Optimal Control. In Mathematics in Science and Engineering. Volume 56. Academic Press, New York; 1969:viii+136.

6. Lakshmikantham V, Bainov DD, Simeonov PS: Theory of Impulsive Differential Equations. World Scientific, Singapore; 1989.

7. Pandit SG: Systems described by differential equations containing impulses: existence and uniqueness. Rev Roum Math Pures Appl Tome 1981, XXVI:879-887.

8. Deimling K: Multivalued Differential Equations.Edited by: W. De Gruyter. Berlin; 1992:

9. Cannon JR: The solution of the heat equation subject to the specification of energy. Quart Appl Math 1963, 21:155-160.

10. Bitsadze AV, Samarski AA: Some generalizations of linear elliptic boundary value problems. Soviet Math Dokl 1969, 10:398-400.

11. Boucherif A: Second order boundary value problems with integral boundary conditions. Nonlinear Anal 2009, 70:364-371.

12. Aubin JP, Cellina A: Differential Inclusions. Springer Verlag, Berlin; 1984.

13. Hu S, Papageorgiou NS: Handbook of Multivalued Analysis, vol. I: Theory. Kluwer, Dordrecht; 2000

14. Belov SA, Chistyakov W: A selection principle for mappings of bounded variation. J Math Anal Appl 2000, 249:351-366

15. Chistyakov W: On the theory of set-valued maps of bounded variation of one variable. Sbornil: Mathematics 1998, 189(5):797-819.

16. Dhage DC: Multivalued mappings and fixed points II. Tamkang J Math 2006, 37:27-46.

17. Agarwal RP, O'Regan D: Set-Valued Mappings with Applications in Nonlinear Analysis. In Series in Mathematical Analysis and Applications. Volume 4. Taylor \& Francis, London; 2002.

18. Chistyakov W, Nowak A: Regular Caratheodory-type selectors under no convexity assumptions. J Funct Anal 2005, 225:247-262.

doi:10.1186/1687-1847-2011-17

Cite this article as: Agarwal and Boucherif: Nonlocal conditions for differential inclusions in the space of functions of bounded variations. Advances in Difference Equations 2011 2011:17. 